ESAIM: PROCEEDINGS, March 2012, Vol. 35, p. 287-292

Fédération Denis Poisson (Orléans-Tours) et E. Trélat (UPMC), Editors

\title{
A FINITE-VOLUME SCHEME FOR A KIDNEY NEPHRON MODEL
}

\author{
Aurélie Edwards ${ }^{1}$, Nicolas Seguin ${ }^{2}$ and Magali Tournus ${ }^{3}$
}

\begin{abstract}
We present a finite volume type scheme to solve a transport nephron model. The model consists in a system of transport equations with specific boundary conditions. The transport velocity is driven by another equation that can undergo sign changes during the transient regime. This is the main difficulty for the numerical resolution. The scheme we propose is based on an explicit resolution and is stable under a CFL condition which does not depend on the stiffness of source terms.

Résumé. Nous présentons un schéma numérique de type volume fini que l'on applique à un modèle de transport dans le néphron. Ce modèle consiste en un système d'équations de transport, avec des conditions aux bords spécifiques. La vitesse du transport est la solution d'un autre système d'équation et peut changer de signe au cours du régime transitoire. Ceci constitue la principale difficulté pour la résolution numérique. Le schéma proposé, basé sur une résolution explicite, est stable sous une condition CFL non restrictive.
\end{abstract}

\section{INTRODUCTION}

The mechanisms by which the kidney can produce very concentrated urine remain partly unclear. Mathematical models have been useful in elucidating the role that the specific renal architecture and the permeability properties of the kidney tubules and blood vessels play in the urine concentrating mechanism. Few of these models account for dynamic changes; in this study, we develop a robust method to solve a dynamic model of fluid and solute transport along nephrons, which are the functional units of the kidney. The nephron is represented as a series of tubes arranged in a counter-current manner, along which water and solutes are exchanged with the interstitium. Transport is driven by convection, diffusion, and energy-consuming pumps, the parameters for which are taken to be time-independent.

We present here a finite volume resolution [2] for a 5-tube dynamic model associated with the stationary model [3] of nephron transport. Our results indicate that this scheme is stable and robust, and that the algorithm relaxes toward a unique stationary state which does not depend on initial conditions. We prove that it preserves the positivity under an explicit CFL condition and ensures mass conservation at equilibrium. We describe the algorithm for a 2-tube model with one solute but it can easily be extended to 5 tubes and to more solutes. Numerical simulation results are shown for the 5-tube model, with one solute.

\footnotetext{
1 UPMC Univ Paris 06, Univ Paris 05, INSERM UMRS872, and CNRS ERL7226, Laboratoire de génomique, physiologie et physiopathologie rénales, Centre de Recherche des Cordeliers, F-75006, Paris

2 UPMC Univ Paris 06, CNRS UMR 7598, Laboratoire Jacques-Louis Lions, F-75005, Paris

${ }^{3}$ UPMC Univ Paris 06, CNRS UMR 7598,Laboratoire Jacques-Louis Lions, F-75005, Paris
}

(C) EDP Sciences, SMAI 2012 


\section{Description of the MOdel}

A minimal representation of a functional kidney unit includes 5 tubes, 3 of which depict one nephron and the other 2 adjacent counter-current blood vessels. In these tubes, fluid circulates with a flow $F$, and solutes are present in concentration $C_{i}, i=1 . . I$.
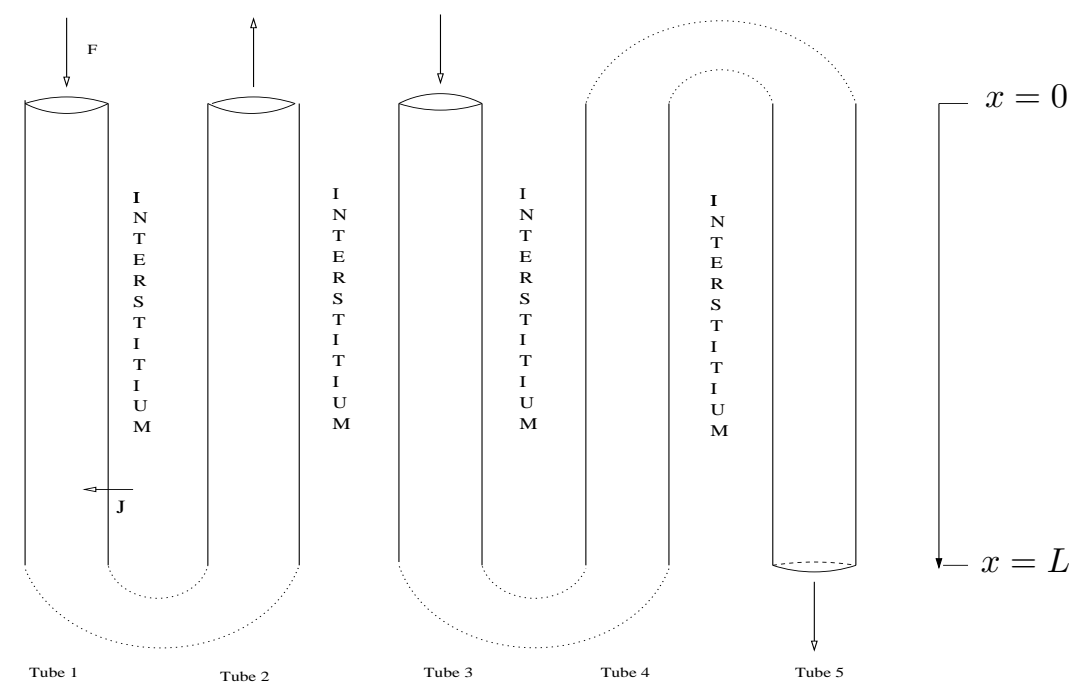

FiguRE 1. Representation of the nephron model. It consists in 5 tubes bathing in a common interstitium.

\subsection{Parameters and variables}

We assume that all parameter values depend only on the depth $x \in[0, L]$ and that the variables depend on $x$ and time $t$. In that sense, our model is one dimensional. Physical parameters are summarized in table 1 The

TABle 1. Parameters depending on $x,(0 \leq x \leq L)$.

\begin{tabular}{|c|c|c|}
\hline \hline Parameter & Description & Unit \\
\hline$R^{j}$ & Radius of tube $j$ & $m$ \\
$L_{P}^{j} R T$ & Water permeability of tube $j$ & $m^{4} \cdot s^{-1} \cdot \mathrm{mol}^{-1}$ \\
$P_{i}^{j}$ & Permeability to solute $i$ of tube $j$ & $m . s^{-1}$ \\
$\sigma_{i}^{j}$ & Reflection coefficient of tube $j$ to solute $i$ & no unit \\
$V_{m}^{j}$ & Rate of active transport across tube $j$ & $m o l . m^{-2} \cdot s^{-1}$ \\
\hline
\end{tabular}

physical variables of the model are as follows:

- $F^{j}(t, x)\left(m^{3} \cdot s^{-1}\right)$ is the water flow in tube $j$ at depth $x$ and time $t$. It represents the volume of water passing through the area centered in $x$ in 1 second.

- $J_{V}^{j}(t, x)\left(m^{2} . s^{-1}\right)$ is the water flux across the wall of tube $j$. It represents the signed surface of water entering tube $j$ at depth $x$ in 1 second.

- $C_{i}^{j}(t, x)\left(\right.$ mol. $\left.m^{-3}\right)$ is the concentration of solute $i$ in tube $j(j=1 . .5$ or $j=i n t)$. The product $F^{j}(t, x) C_{i}^{j}(t, x)\left(\right.$ mol.s $\left.{ }^{-1}\right)$ represents the number of moles of solute $i$ passing through the area centered in $x$ in 1 second.

- $J_{i}^{j}(t, x)\left(\right.$ mol. $\left.m^{-1} . s^{-1}\right)$ is the flux of solute $i$ entering tube $j(j=1 . .5, i n t)$. It represents the number of moles entering tube $j$ at depth $x$ in 1 second. 
ESAIM: PROCEEDINGS

\subsection{The system}

These variables are related by the equations of water conservation and solute conservation in the tubes and in the interstitium for $0 \leq x \leq L$

$$
\left\{\begin{aligned}
\frac{\partial}{\partial x} F_{V}^{j}(t, x) & =J_{V}^{j}(t, x), & & j=1, \ldots, 5, \\
\pi\left(R^{j}\right)^{2} \frac{\partial}{\partial t} C_{i}^{j}(t, x)+\frac{\partial}{\partial x}\left(F_{V}^{j}(t, x) C_{i}^{j}(t, x)\right) & =J_{i}^{j}(t, x), & & j=1, \ldots, 5, \quad i=1, \ldots, I, \\
\pi\left(R^{i n t}\right)^{2} \frac{\partial}{\partial t} C_{i}^{\text {int }}(t, x) & =J_{i}^{\text {int }}(t, x), & & i=1, \ldots, I
\end{aligned}\right.
$$

with water fluxes given by

$$
J_{V}^{j}(t, x)=2 \pi R^{j}(x) L_{P}^{j}(x) R T \sum_{i=1}^{I} \sigma_{i}^{j}(x)\left(C_{i}^{j}(t, x)-C_{i}^{i n t}(t, x)\right), \quad j=1,3,4,5,
$$

There is a specific difficulty for tube 2 because the water permeability of its wall is so large that it cannot be measured ( $L_{P}^{2}$ is unknown). Thus, we compute the water flux entering tube 2 using the hypothesis of a rigid interstitium, which means that there is no water accumulation therein. Thus we determine $J_{V}^{2}$ by

$$
\sum_{j=1}^{5} J_{V}^{j}(t, x)=0 \quad \forall t>0, \quad \forall x \in[0, L]
$$

and the solute fluxes are given by

$$
J_{i}^{j}(t, x)=-2 \pi R^{j}(x) P_{i}^{j}(x)\left(C_{i}^{j}(t, x)-C_{i}^{i n t}(t, x)\right)+J_{V}^{j}(t, x)\left(1-\sigma_{i}^{j}(x)\right) C_{i}^{\alpha}(t, x)-2 \pi R^{j} V_{m}^{j} \frac{C_{i}^{j}}{K_{m}^{j}+C_{i}^{j}},
$$

with

$$
C_{i}^{\alpha}(t, x)= \begin{cases}C_{i}^{i n t}(t, x) & \text { for } J_{V}^{j}(t, x)>0 \\ C_{i}^{j}(t, x) & \text { for } J_{V}^{j}(t, x) \leq 0\end{cases}
$$

By conservation of each solute we have the relation

$$
J_{i}^{i n t}(t, x)=-\sum_{j} J_{i}^{j}(t, x) \quad \forall t>0, \forall x \in[0, L] .
$$

\subsection{The boundary conditions}

The tube architecture and the water flow direction (see figure 1) enable us to impose the following boundary conditions

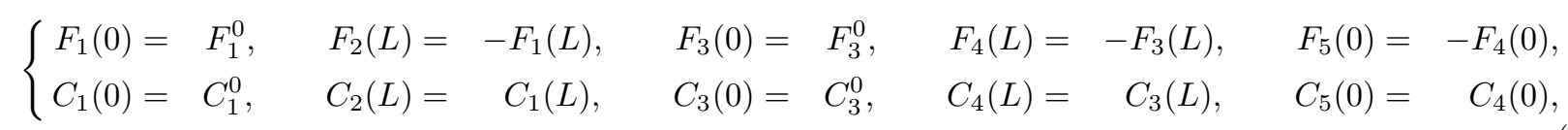

where $F_{1}^{0}, F_{3}^{0}, C_{1}^{0}, C_{3}^{0}$ are four nonnegative given values. It has been proved [8] that a simplified model relaxes toward a unique stationary state for any initial conditions. We expect this to also be the case for the model at hand. 


\section{Numerical RESOlution}

Several numerical studies of similar dynamic systems have been published. A first issue was raised by Moore and Marsh [7] concerning the instability of usual schemes for realistic parameter values. Indeed, the high permeability to water in some tube segments can result in very large water movements across those segments. If enough water is extracted from the tube, water flow can reverse direction during the dynamic process thereby rendering the numerical method unstable. The method they were using did not deal with this problem and forced them to consider lower values of the water permeability $L_{P}$. To avoid numerical instabilities arising from reversal of flow, new dynamic numerical methods were implemented, invoving computation of space derivatives upwind along the flow direction. An explicit method for solving hyperbolic PDEs [5] [6] was adapted by Layton and Pitman, but the CFL condition they obtained was tough. To deal with this problem, a semi lagrangian-semi implicit (SLSI) method was implemented [1]. The drawback of this method is the lack of accuracy, thus it is combined with a Newton-type solver, which uses the solution from the SLSI method as an initial guess. More recently, a scheme based on the second order Godunov method was developed [4] for a 3-tube model whose particularity is that the transmural solute flux contains no convective term.

We present here a scheme for the more extensive model, which deals with transient reversal flows and which converges for any initial conditions and for realistic parameter values under an explicit CFL condition.

\subsection{The numerical scheme}

We propose here a finite-volume scheme, combined with a splitting method. To simplify the notations, we write the scheme for only two tubes (tubes 1 and 2), but it is easy to infer the scheme for the 5 tubes. As tube 2 plays a specific part, we keep it in our minimal scheme. With only two tubes, we could have simplified the scheme, for example by directly imposing $F_{k}^{2, n}=-F_{k}^{1, n}$ but this is not possible in the complete model and thus we do not make this simplification. We also present the scheme with one solute $(I=1)$ but it is easily generalized to $I$ solutes, the only differences lying in the computation of water fluxes (which then contain one more term).

For the initial condition, we define the cell averages

$$
C_{k}^{j, 0}=\frac{1}{\Delta x} \int_{Q_{k}} C^{j}(x, 0) d x, \quad j=1,2, \quad k=1, \ldots, N .
$$

We discretize the parameters $P$ as

$$
P_{k}^{j}=\frac{1}{\Delta x} \int_{Q_{k}} P^{j}(x) d x, \quad j=1,2, \quad k=1, \ldots, N .
$$

We use a mesh size $\Delta x=L / N$ where $N$ is the number of cells $Q_{k}=\left(x_{k-1 / 2}, x_{k+1 / 2}\right), C_{k}^{\alpha, n}, \alpha=1,2$, int represents the value of the concentration in cell $k$ after $n$ iterations, and we impose at the outlet $C_{0}^{1, n}=$ $C^{1}(0, t), F_{0}^{1, n}=F^{1}(0, t), C_{N+1}^{2, n}=C_{N}^{1, n}$ and $F_{N+1}^{2, n}=-F_{N}^{1, n}$. The outlet of tube 2 is open, we have to define the values $C_{0}^{2, n}$ and $F_{0}^{2, n}$. We choose $C_{0}^{2, n}=C_{1}^{2, n}$ and $F_{0}^{2, n}=F_{1}^{2, n}$, and we will so do for every open tube outlet in the complete scheme. We first compute the discrete water fluxes and flows

$$
\left\{\begin{array} { l } 
{ J _ { V , k } ^ { 1 , n } = 2 \pi R _ { k } ^ { 1 } L _ { p , k } ^ { 1 } \sigma _ { k } ^ { 1 } ( C _ { k } ^ { 1 , n } - C _ { k } ^ { i n t , n } ) , } \\
{ J _ { V , k } ^ { 2 , n } = - J _ { V , k } ^ { 1 , n } }
\end{array} \quad \left\{\begin{array}{l}
\frac{F_{k}^{1, n}-F_{k-1}^{1, n}}{\Delta x}=J_{V, k}^{1, n}, \quad F_{N+1}^{2, n}=-F_{N}^{1, n}, \\
\frac{F_{k}^{2, n}-F_{k+1}^{2, n}}{\Delta x}=J_{V, k}^{2, n} .
\end{array}\right.\right.
$$

We then define the numerical flux of water between meshes $k$ and $k+1$ as

$$
F_{k+\frac{1}{2}}^{j, n}=\frac{1}{2}\left(F_{k}^{j, n}+F_{k+1}^{j, n}\right)
$$


Other interpolations at the cells interfaces are possible but we observe experimentally that the choice of $F_{k+\frac{1}{2}}^{j, n}$ does not affect the numerical solution.

The concentration in each mesh is updated with a splitting method in two steps:

First step A provisional concentration is obtained using the axial convection part of the equation, then, the provisional concentration is updated thanks to the transmural solute fluxes. To compute the provisional concentration, we write the scheme under a conservative form

$$
\left(R_{k}^{j}\right)^{2} \frac{C_{k}^{j, n+\frac{1}{2}}-C_{k}^{j, n}}{\Delta t}+\frac{1}{\Delta x}\left(g_{k+\frac{1}{2}}^{j, n}-g_{k-\frac{1}{2}}^{j, n}\right)=0, \quad j=1,2
$$

where $g_{k-\frac{1}{2}}^{i, n}$ is the numerical flux from the mesh $k-1$ to the mesh $k$ in tube $i$ after $n$ iterations. We upwind the numerical fluxes as

$$
g_{k+\frac{1}{2}}^{i, n}= \begin{cases}F_{k+\frac{1}{2}}^{j, n} C_{k}^{j, n} & \text { if } F_{k+\frac{1}{2}}^{j, n}>0 \\ F_{k+\frac{1}{2}}^{j, n} C_{k+1}^{j, n} & \text { otherwise }\end{cases}
$$

This definition ensures mass-conservation from the mesh $N$ of tube 1 to the mesh $N$ of tube 2 .

Second step To compute the definitive value of the concentration, we use a semi-implicit scheme so as to avoid limitations on the CFL condition,

$$
\left(R_{k}^{j}\right)^{2} \frac{C_{k}^{j, n+1}-C_{k}^{j, n+\frac{1}{2}}}{\Delta t}=J_{i, k}^{j, n}
$$

with

$$
J_{i, k}^{j, n}=\left\{\begin{array}{l}
-2 \pi R_{k}^{j} P_{k}^{j}\left(C_{k}^{j, n+1}-C_{k}^{i n t, n}\right)+J_{V, k}^{n}\left(1-\sigma_{k}^{j}\right) C_{k}^{i n t, n}-V_{m, k}^{j} R_{k}^{j} \frac{C_{k}^{j, n+1}}{1+C_{k}^{j, n+\frac{1}{2}}} \quad \text { if } J_{V, k}^{j, n}>0 \\
-2 \pi R_{k}^{j} P_{k}\left(C_{k}^{j, n+1}-C_{k}^{i n t, n}\right)+J_{V, k}^{j, n}\left(1-\sigma_{k}^{j}\right) C_{k}^{j, n+1}-V_{m, k}^{j} R_{k}^{j} \frac{C_{k}^{j, n+1}}{1+C_{k}^{j, n+\frac{1}{2}}} \quad \text { if } J_{V, k}^{j, n}<0
\end{array}\right.
$$

We can easily invert this implicit formulation and express $C_{k}^{j, n+1}$ in function of $C_{k}^{n+\frac{1}{2}}$ and $C_{k}^{i n t, n}$. Then we calculate

$$
\begin{gathered}
J_{k}^{i n t, n}=-J_{k}^{1, n}-J_{k}^{2, n} \\
\left(R_{k}^{i n t}\right)^{2} \frac{C_{k}^{i n t, n+1}-C_{k}^{i n t, n}}{\Delta t}=J_{k}^{i n t, n} .
\end{gathered}
$$

\subsection{Properties of the numerical scheme}

The scheme preserves the positivity under the CFL condition We want to guarantee the property

$$
\left(C_{k}^{j, n}\right)_{k \in[1, N]} \geq 0 \Longrightarrow\left(C_{k}^{j, n+1}\right)_{k \in[1, N]} \geq 0
$$

In equation 12 by distinguishing 4 cases depending on the sign of $F_{k}^{j, n}$ and $F_{k-1}^{j, n}$, we show that the CFL condition can be written

$$
\Delta t \leq \min _{j=1,2}\left\{\frac{\Delta x}{2} \frac{\min _{k}\left(R_{k}^{j}\right)^{2}}{\max _{k}\left|F_{k}^{j}\right|}\right\}
$$

There is no extra CFL condition coming from the treatment of source terms because we use an implicit scheme. To accelerate the convergence, we compute a new optimal $\Delta t$ at each time step. 
At equilibrium, there is mass conservation At equilibrium, the quantity $\sum_{j=1}^{2} g_{k+\frac{1}{2}}^{j, n}$ does not depend on $k$. This property represents the mass conservation at a discrete level and can be used as a criterium to determine when the system has reached equilibrium.

\subsection{Numerical illustrations}

We are interested in the profiles of flows and concentrations at equilibrium for the 5 -tube model. We run the algorithm until $\sum_{j=1}^{5} g_{k+\frac{1}{2}}^{j, n}<10^{-12}$ which corresponds to $T=200$. For the numerical simulation, we use realistic parameters and boundary conditions as found in [3].
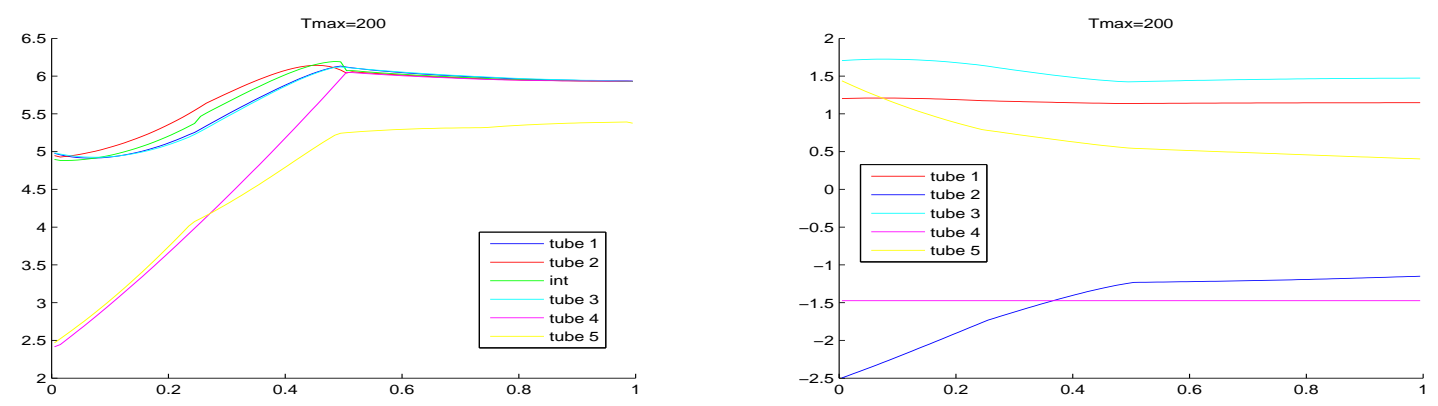

Figure 2. Profiles of water flows (on the right) and concentrations (on the left) at equilibrium. We chose $C_{0}^{1}=C_{0}^{2}=5, F_{0}^{1}=1.2, F_{0}^{3}=1.7$. The profiles do not depend on the initial condition.

We observe that solute concentration increases on $\left[0, \frac{L}{2}\right]$ and remains approximately constant on $\left[\frac{L}{2}, L\right]$, This profile is to be expected physiologically because $V_{m}(x)$ (i.e the active pumping of solute) is almost equal to zero everywhere except in tube 4 between 0 and $\frac{L}{2}$.

Acknowledgments. Funding for this study was provided by the program EMERGENCE (EME 0918) of the Université Pierre et Marie Curie (Paris Univ. 6).

\section{REFERENCES}

[1] H.E. Layton A.T. Layton. A semi-Lagrangian semi-implicit numerical method for models of the urine concentrating mechanism. SIAM J. Sci. Comput.

[2] F.Bouchut. Non linear stability of finite volume methods for hyperbolic conservation laws and well balanced schemes for sources. Birkhaüser-Verlag, 2004.

[3] S. Hervy and S.R. Thomas. Inner medullary lactate production and urine-concentrating mechanism: a flat medullary model. Am.J.Physiol, (284):F65-F81, 2002.

[4] A.T. Layton. A methodology for tracking solute distribution in a mathematical model of the urine concentrating mechanism. Journal of Biological System, 13(4):1-21, 2005.

[5] H. Layton and E. Pitman. A dynamic numerical method for models of renal tubules. Bulletin of Mathematical Biology, 56:547$565,1994$.

[6] H. E. Layton, E. Bruce Pitman, and Mark A. Knepper. A dynamic numerical method for models of the urine concentrating mechanism. SIAM J. Appl. Math., 55:1390-1418, October 1995.

[7] L. C. Moore and D. J .Marsh. How descending limb of henle's loop permeability affects hypertonic urine formation. Am.J.Physiol, (239):F57-F71, 1980.

[8] M. Tournus, A. Edwards, N. Seguin, and B. Perthame. Analysis of a simplified model of the urine concentration mechanism. Preprint, 2011. 